\title{
A New Approach to Verification of Catch Reports Based on the Classification of Satellite Positioning Tracks in the Fisheries Monitoring System
}

\author{
Vladimir N. Pyrkov ${ }^{1}$, Petr M. Vasilets ${ }^{2}$, Alexander Yu. Degay ${ }^{1}$, Mikhail V. Andreev ${ }^{1}$, Vladimir N. \\ Chernykh ${ }^{1}$, Andrey V. Solodilov ${ }^{1}$, \\ ${ }^{1}$ Space Research Institute Academy of Sciences, Moscow, Russia \\ vpyrkov@mail.ru \\ ${ }^{2}$ Kamchatka Research Institute of Fisheries and Oceanography, Petropavlovsk-Kamchatsky, Russia \\ vasilets@kamniro.ru
}

\begin{abstract}
The report develops a method of verification of fishing reports in the fisheries monitoring system based on comparison with the satellite positioning of ships. The method is based on automatic classification of vessel tracks with reference to trawling periods to fishing zones. Further, according to the data obtained in the previous step, the fishing effort per day in the fishing zone is estimated. Then the fishing effort is compared with the catch in the area per day. The efficiency of the above proposed method for the detection of gross distortions of the reporting of catch in the ship's daily reports is shown.
\end{abstract}

Keywords: algorithm of automatic classification of tracks, fishery monitoring system, positional satellite data, verification of multi-parameter reporting 


\title{
НОВЫЙ ПОДХОД К ВЕРИФИКАЦИИ ОТЧЕТОВ О ВЫЛОВЕ НА ОСНОВЕ КЛАССИФИКАЦИИ ТРЕКОВ СПУТНИКОВОГО ПОЗИЦИОНИРОВАНИЯ В СИСТЕМЕ МОНИТОРИНГА РЫБОЛОВСТВА
}

\author{
В.Н. Пьрков ${ }^{1}$, П.М. Василей ${ }^{2}$, А.Ю. Дегай ${ }^{1}$, М.В. Андреев ${ }^{1}$, В.Н. Черных ${ }^{1}$, А.В. Солодилов ${ }^{1}$ \\ ${ }^{1}$ Институт космических исследований РАН, Москва, Россия \\ vpyrkov@mail.ru \\ 2 Камчатский научно-исследовательский институт рыбного хозяйства и океанографии, \\ Петропавловск-Камчатский \\ vasilets@kamniro.ru
}

\begin{abstract}
В докладе развивается методика верификации рыбопромысловых отчетов в системе мониторинга рыболовства на основе сопоставления с данными спутникового позиционирования судов. Методика основывается на автоматической классификации треков судна с привязкой периодов траления к промысловым зонам. Далее по полученным на предыдущем шаге данным оценивается промысловое усилие за сутки в рыбопромысловой зоне. Затем производится сравнение промысловых усилий с уловом в районе в сутки. Показана эффективность выше предложенной методики для выявления грубых искажений отчетности о вылове в судовых суточных донесениях.
\end{abstract}

Ключевые слова: алгоритм автоматической классификации треков, отраслевая система мониторинга водных биологических ресурсов, наблюдения и контроля за деятельностью промысловых судов (ОСМ), позиционные спутниковые данные, верификация многопараметрической отчетности.

\section{Введение}

Достоверность отчетов о вылове в отраслевой системе мониторинга рыболовства (ОСМ) является важным фактором при принятии как стратегических решений, например таких как определение общедопустимого вылова в различных промысловых зонах так и для решения текущих задач, например определения соответствия вылова судном и выданных данному судну разрешений на добычу(вылов). Ранее на некоторых примерах было показано [Василец и др., 2017], что при визуальной классификации треков судна по данным спутникового позиционирования можно выявить явные противоречия в судовых суточных донесениях. В данном докладе развивается и оценивается эффективность методики верификации промысловой отчетности на основе сопоставления с данными спутникового позиционирования судов. Приведенный в работе [Василец и др., 2017] анализ позволяет сделать вывод о необходимости разработки и внедрения в ОСМ дополнительных методик контроля информации о вылове судна. В качестве одного из таких методов контроля данных ССД может быть предложена "видеофиксация" уловов, при этом необходимо отметить, что реальных отечественных систем спутниковой связи для оперативного востребования данной информации в настоящее время не 
существует. С другой стороны, могут быть предприняты попытки автоматизировать рассмотренный в работе [Василец и др., 2017] метод комплексного анализа позиционной и промысловой информации, включающий в себя классификацию фрагментов трека судна при достаточно высокой частоте позиционного опроса. В работе [Василец и др., 2018] , был предложены основные этапы метода выявления подозрительной отчетности о вылове, в том числе, автоматическая классификация трека судна с целью выделить фрагменты, когда судно проводит операции по добыче вылову; по координатам определенных на предыдущем этапе фрагментов определяются промысловые зоны; далее оценивается время промысловых усилий в каждой из отчетных промысловых зон; определяется отношение вылова к времени затраченному на промысел в каждой из анализируемых промысловых зон; по отклонению вышеуказанного отношения для судна от среднего для промысловой зоны и сезона добычи (вылова), то делается вывод достоверности отчетности рассматриваемого судна. Таким образом, внедрение предложенной методики основывается на развитии автоматической классификации треков судна [Дегай и др., 2017] с привязкой периодов траления к промысловым зонам. Возможность автоматической классификации треков судов повысилась благодаря достаточно высокой частоте позиционного опроса судна. Использование терминалов АИС в составе судового технического средства контроля ОСМ позволило с помощью спутниковой составляющей системы мониторинга ориентировочно в семь с половиной раз увеличить частоту спутникового позиционного опроса судна.

\section{Методика}

Для конкретной реализации методики верификации данных была предпринята попытка выполнить последовательность действий приведенных в таблице 1.

Таблица 1. Последовательность этапов определения обобщенного отношения вылова к промысловому усилию в зависимости от сезона, промысловой зоны и вида водного биологического ресурса

\begin{tabular}{|c|c|}
\hline пП & Описание этапа \\
\hline 0 & $\begin{array}{l}\text { Входные параметры список судов ves_list тип модели model дата date } \\
\text { далее все сущности являются функциями date и model (если специально не оговорено) }\end{array}$ \\
\hline 1 & $\begin{array}{l}\text { date_begin }=\text { date }- \text { dtwindow date_end }=\text { date }+ \text { dtwindow } \\
\text { datetime_start=date_begin }+ \text { timeshift } \\
\text { datetime_finish=date_end }+ \text { timeshift } \\
\text { dtwindow timeshift зависят только от модели }\end{array}$ \\
\hline 2 & catch_sum - полный вылов по списку судов с date_begin до date_end \\
\hline 3 & $\begin{array}{l}\text { catch_fish_region(ves,fish,region)* } \\
\text { ves_trawl_list, fish_list, region_list" } \\
\text { * входят данные, только такие, для которых вылов больше чем }>\text { catch_sum } * 10^{\text {-ncatch_power }} \\
\text { ncatch_power определяется моделью }\end{array}$ \\
\hline 4 & $\begin{array}{l}\text { track_n(ves) } \\
\text { latitude(ves,track) longitude(ves,track) } \\
\text { trawl_time(ves,track) } \\
\text { full_trawl_time_ves(ves) }=\sum_{\text {track }} \text { trawl_time(ves,track) }\end{array}$ \\
\hline 5 & $\begin{array}{l}\text { region(ves,track) определяется latitude(ves,track) longitude(ves,track) широтой, долготой трека, и } \\
\text { полигоном промысловой зоны region polygon }\end{array}$ \\
\hline 6 & $\begin{array}{lccc}\text { catch_fish_track(ves,track,fish) } & \text { определяется } & \text { по } & \text { catch_fish_region(ves,fish,region) } \\
\text { region(ves,track) (п5), trawl_time(ves,track), full_trawl_time_ves(ves) }(\text { (п4) } & \text { (п3), }\end{array}$ \\
\hline 7.0 & Возможна выгрузка в таблицу построения полей вылова catch_field_locate по следующим данным \\
\hline
\end{tabular}




\begin{tabular}{|c|c|}
\hline \multirow{4}{*}{7.1} & catch_fish_track(ves,track,fish), latitude(ves,track) longitude(ves,track), date, model \\
\hline & $\begin{array}{r}\text { Наконец определяем обобщенные параметры вылова, промыслового времени и соотношение } \\
\text { Generalised_fish_region_catch (date,model,fish,region) }=\sum \text { catch_fish_track(ves,track,fish) } \\
\text { ves, region(ves,track) }=\text { region }\end{array}$ \\
\hline & $\begin{array}{r}\text { Generalised_region_trawl_time (date,model,region) }=\sum \text { trawl_time(ves,track) } \\
\text { ves, region(ves,track) }=\text { region } \\
\text { catch_fish_track }(\mathrm{ves}, \operatorname{track}, f i s h)>\mathbf{0}\end{array}$ \\
\hline & $\begin{array}{l}\text { Generalised_fish_region_ratio(date,model,fish,region)= } \\
\text { Generalised_fish_region_catch/Generalised_fish_region_trawl_time }\end{array}$ \\
\hline
\end{tabular}

В соответствии с предложенной последовательностью действий был выбран список из семи судов одного типа - СРТМ (Средний рыболовный траулер морозильный). Данные суда осуществляли добычу (вылов) главным образом трески и камбалы донным тралом в феврале 2018 года в Карагинской подзоне и Западно-Беринговоморской зоне. Для выделения периодов траления был выбран простейший подход - считалось, что судно проводит траловые работы, если его скорость больше двух и меньше пяти узлов. Затем в данных промежутках проводилась выборка из базы данных ОСМ координат и времени судна. Далее по координатам соответствующим треку определяется, к какой промысловой зоне данный трек относится.

Разница между окончанием и началом трека траления просуммированная за сутки дает дневное усилие судна для промысловой зоны. Таким образом, выполнены 4 и 5 пункты таблицы 1.

Результаты. При попытках перейти к 6 и 7 пунктам Таблицы 1 обнаружены проблемы. Не каждому дневному промысловому усилию, находилось соответствие в отчетах по вылову в судовых суточных донесениях, что подтверждено примерами визуального анализа на ниже представленном рисунке 1 .

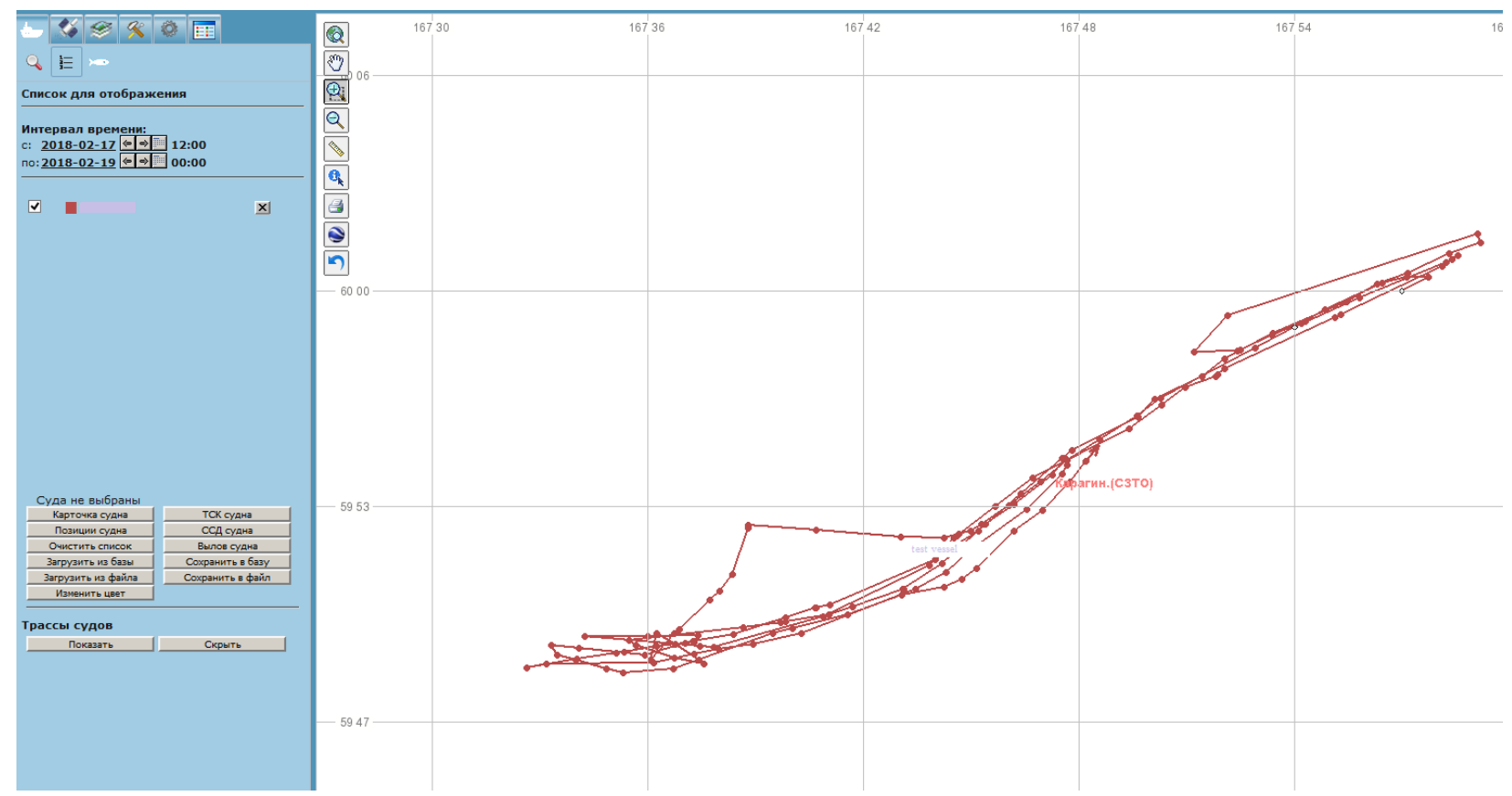

Рисунок 1. Пример искажения отчетности за 18 февраля. Судно совершает маневры характерные для траловых работ, что подтверждает результат автоматической классификации трека по спутниковым позиционным данным, а декларирует отсутствие вылова

В то же время не каждому дневному вылову находилось соответствие дневного промыслового усилия. Например, по отчетам судно декларирует вылов в Западно-Беринговоморской зоне, в то время как простейшая автоматическая классификация по спутниковым позиционным данным определяет работу тралом в Карагинской подзоне. Был проведен визуальный анализ треков 
судна за 4 и 17 февраля. Визуальный анализ подтвердил результаты простейшей автоматической классификации.

Заключение. В результате многочисленных искажений отчетности о вылове анализ отношения вылова к промысловым усилиям промысловой зоны, вида биоресурсов, типа судна и сезона лова, на выбранном списке судов оказался затруднительным. В то время как даже простейший алгоритм автоматической классификации трека судна с последующей привязкой тралового фрагмента трека к промысловой зоне показал свою эффективность для выявления грубых искажений отчетности о вылове в судовых суточных донесениях.

Работы проводились с использованием технологий и данных изентра коллективного пользования ЦКП "ИКИ-Мониторинг" [Лупян и др., 2015].

Работа выполнена при поддержке ФАНО (тема "Мониторинг", госрегистрация № 01.20.0.2.00164).

\section{References}

[1] Vasilets P.M., Terentyev D.A., Pyrkov V.N., Analysis of spatial distribution of flounder catches in the waters adjacent and off kamchatka for 2011-2016 on the data of the Fisheries Sectoral Monitoring System, Issledovaniya vodnykh biologicheskikh resursov Kamchatki i severo-zapadnoi chasti Tikhogo okeana, 2017, 47, pp. 65-77. DOI:10.15853/2072-8212.2017.47.65-76. (In Russian).

[2] Vasilets P.M., Terentyev D.A.,Korobov S.A., Pyrkov V.N., Solodilov A.N., Degay A.Yu., Possibility for assessing the reliability of spatial information on bottom fish catch in Fishery Monitoring System on the basis of an integrated analysis of daily fishing reporting and satellite positioning data of fishing vessels, Sovremennye problemy distantsionnogo zondirovaniya Zemli iz kosmosa, 2018., 15 (7), pp. 35-42. DOI: 10.21046/2070-74012018-15-7-35-42. (In Russian).

[3] Degay A.Yu., Pyrkov V.N., ChernykhN.N., Solodilov A.N., Consideration of the possibility of classification of vessel tracks in the fisheries monitoring system using modern technical means of control, 15 konferentsiya 'Sovremennye problemy distantsionnogo zondirovaniya Zemli iz kosmosa"' Moscow, 13-17 November 2017, Book of Abstracts,2017, p.82. (In Russian).

[4] Loupian E.A., Proshin A.A., Burtsev M.A., Balashov I.V., Bartalev S.A., Efremov V.Yu., Kashnitskiy A.V., Mazurov A.A., Matveev, A.M. Sudneva O.A., Sychugov I.G., Tolpin V.A., Uvarov I.A., IKI center for collective use of satellite data archiving, processing and analysis systems aimed at solving the problems of environmental study and monitoring, Sovremennye problemy distantsionnogo zondirovaniya Zemli iz kosmosa, 2015., 12 (5), pp.263-284. (In Russian). 